\title{
3.4/4.0 GHz Tunable Resonant Cavity in SIW Technology Using Metal Post and PIN Diode on a Low-Cost Biasing Network for $5 \mathrm{G}$ Applications
}

\author{
Ricardo Caranicola Caleffo ${ }^{1}$ (D), Fatima Salete Correra ${ }^{1}$ (C) \\ ${ }^{1}$ Universidade de São Paulo (USP), Escola Politécnica, Departamento de Engenharia de Sistemas Eletrônicos \\ Av. Prof. Luciano Gualberto, Trav. 3, $n^{\circ}$ 380, SP - São Paulo, Brazil \\ ricardocaleffo@usp.br,fcorrera@lme.usp.br
}

\begin{abstract}
This paper presents a dual-band resonant cavity in Substrate Integrated Waveguide (SIW) technology to operate in the range from $3.3 \mathrm{GHz}$ to $4.2 \mathrm{GHz}$, spectrum considered for the Fifth Generation (5G) network. The cavity was designed to operate at 3.4 GHz and 4.0 GHz. To achieve different states, a jumper and a PIN diode switch are considered as switching elements, connecting and disconnecting the bottom and the upper walls of the SIW resonant cavity through a metal post inserted inside its internal volume. A fitting equation is proposed to predict the maximum resonance frequency caused by the insertion of a single metal post inside the internal volume of the SIW resonant cavity and a new low-cost biasing network is designed on a thin dielectric substrate allocated on the upper wall of the SIW resonant cavity, using transmissions lines and a single capacitor, reducing the final cost and the insertion losses. Good agreement was observed among the fitting equation, computational electromagnetic simulations, and experimental results, validating the proposed methods.
\end{abstract}

Index Terms - Biasing Network, SIW technology, Tunable circuits, 5G frequencies.

\section{INTRODUCTION}

Industry 4.0 is a new concept being widely explored and considered in many companies and institutions of the world due to the impact on the manufacturing and automation technologies. This new term is also referred to as the fourth industrial revolution and considers the insertion of smart systems in the manufacturing and automation processes aiming to increase productivity and to reduce losses, thus improving capitalization. Smart manufacturing is completely changing the production cycle of industries specialized in different kinds of products and goods. In this new concept, machines are equipped with communication systems, sensors, and actuators; all these components are connected to each other, generating a large amount of collected data to be processed. The emerging concept of Industry 4.0 employs technologies such as the Internet of Things (IoT), Big Data, Artificial Intelligence, and computational simulations [1]-[4].

The IoT is a recent technological diffusion that aims to connect billions of smart objects on the internet. These objects are expected to have network connectivity through an Internet Protocol, allowing them to transmit and receive data via internet networks. Several challenges need to be faced Brazilian Microwave and Optoelectronics Society-SBMO received 6 Sept 2019; for review 19 Sept 2019; accepted 20 Jan 2020 
for the IoT, such as data reliability and communication performance due to a huge quantity of objects connected [5]-[6].

To improve the performance of the smart communication systems used in Industry 4.0, IoT and to accommodate the explosive growth of data traffic, the Fifth Generation $(5 \mathrm{G})$ network is being widely developed and explored, expected to be launched around 2020. The 5G technology is expected to offer data rate above 1 Gbps in a local area network and 500 Mbps in a wide area network, uploading and downloading data much faster than $3 \mathrm{G}$ and $4 \mathrm{G}$ technologies. The $5 \mathrm{G}$ technology also has the task to support massive Multiple Inputs and Multiple Output (MIMO) systems, broadcasting multiple links and increasing the transmission capacity, an essential factor of wireless communication systems. The operating frequencies of the $5 \mathrm{G}$ technology range from $3 \mathrm{GHz}$ to $300 \mathrm{GHz}$ [7]-[10]. The spectrum below $6 \mathrm{GHz}$ ranging from $3.3 \mathrm{GHz}$ to $4.2 \mathrm{GHz}$ is receiving significant attention in various countries and regions from Asia, Europe and the United States of America. Taking into account the global momentum, the Third Generation Partnership Project (3GPP) has been developing technical specifications for the range from $3.3 \mathrm{GHz}$ to $4.2 \mathrm{GHz}[10]$.

A good option to design and to fabricate circuits and components to operate from $3 \mathrm{GHz}$ is the Substrate Integrated Waveguide (SIW) technology due to the low-cost fabrication process and easy integration with planar circuits. The SIW technology allows designing and fabricating planar structures considering a low-loss dielectric substrate whereby the sidewalls of the traditional Rectangular Waveguides (RWG) are replaced by periodical metalized vias, confining the electromagnetic wave within the dielectric substrate [11]-[16]. Due to the good characteristics of the SIW technology, various high-frequency structures are being designed, such as tunable filters [17][18] and liquids electrical characterization sensors [19], demonstrating the high applicability of this microwave technology.

In this context, a tunable resonant cavity in SIW technology using a single metal post and a PIN diode switch is proposed to operate at $3.4 \mathrm{GHz}$ and $4.0 \mathrm{GHz}$, where the range from $3.3 \mathrm{GHz}$ to 4.2 GHz is considered for the $5 \mathrm{G}$ network. Receivers and transmitters to be used in $5 \mathrm{G}$ networks require narrowband filters in the bands below $6 \mathrm{GHz}$ and the designed dual-band resonant cavity can be applied. To obtain multiple states and, consequently, a variation of the resonance frequency, a jumper and a PIN diode switch are used as switching elements, connecting and disconnecting the bottom and the upper wall of the SIW resonant cavity through a single metal post inserted inside its internal volume. A fitting equation is proposed to predict the maximum resonance frequency caused by the insertion of a single metal post inside the internal volume of the resonant cavity and a new lowcost biasing network is also proposed, using transmissions lines and a single capacitor, leading to a low fabrication cost and low insertion losses in comparison with works presented in the literature. 
In the remainder of this paper, Section 2 presents the design considerations, proposing a fitting equation and presenting the design of a tunable resonant cavity and including its biasing network. Section 3 presents the fabricated tunable resonant cavity and the experimental results. Section 4 presents the conclusions. Section 5 shows the acknowledgments and Section 6 shows the references.

\section{DESIGN OF A TUNABLE RESONANT CAVITY IN SIW TECHNOLOGY}

This section presents the design of a SIW resonant cavity operating in $\mathrm{TE}_{101}$ mode, perturbed by the insertion of a metal post through its internal volume, as shown in Fig. 1.

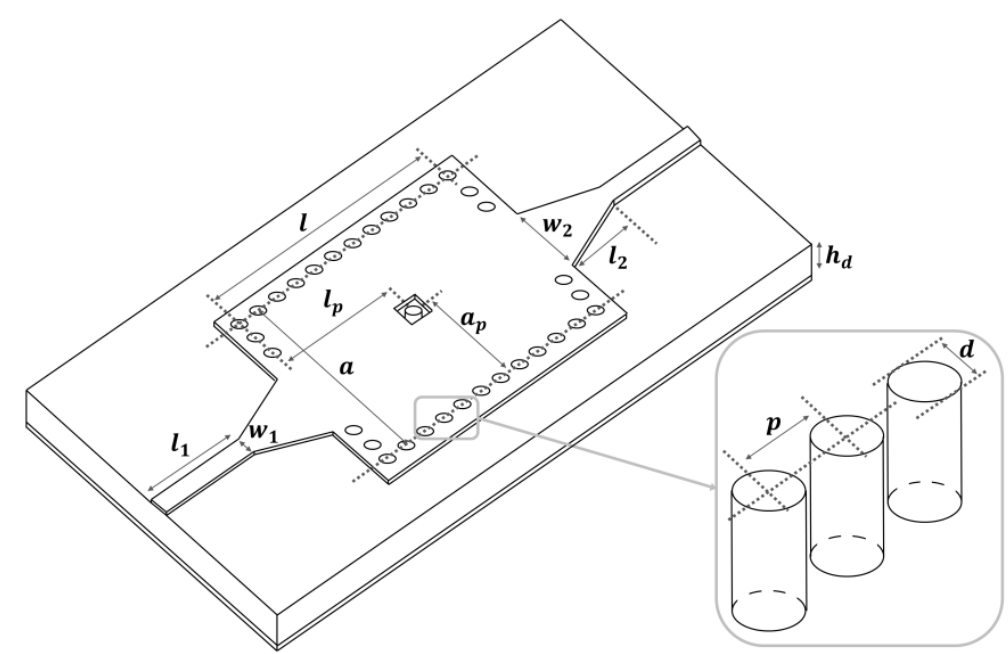

Fig.1. Geometric parameters of the tunable resonant cavity.

The metal post is permanently connected at the bottom wall of the resonant cavity, being connected and disconnected at the upper wall, resulting in two operating states, with two different resonance frequencies.

As shown in [18] and [20], when the metal post is disconnected from the cavity upper wall, the resonance frequency of this resonant cavity is close to the resonance frequency of the unperturbed cavity. The maximum resonance frequency is achieved when the metal post is connected at the upper wall of the cavity and located at the cavity center, where the electrical field of the $\mathrm{TE}_{101}$ mode has its maximum value. The resonance frequency of the cavity can be tuned between these two frequency limits locating the metal post out of the resonant cavity center. It is important to know the maximum resonance frequency of a perturbed resonant cavity because it imposes limits on the design of reconfigurable SIW filters. With this motivation, an analytical equation is proposed to estimate the maximum resonance frequency of a SIW resonant cavity when perturbed by a single metal via through its internal volume, connecting its upper and bottom metal layers.

A jumper and a PIN diode switch are used as switches to connect and to disconnect the upper and bottom walls of the cavity, as used in [18]. A new biasing network employing transmission lines and lumped components is used to bias the PIN diode, resulting in an easy-to-assemble and low-cost solution. 


\section{A. Single metal post in a SIW resonant cavity}

The operation of a resonant cavity is affected by the presence of metallic shapes inside its internal volume that connect the upper and the bottom walls. Aiming to study this phenomenon, a SIW resonant cavity is represented by the rectangular waveguide resonant cavity shown in Fig. 2,

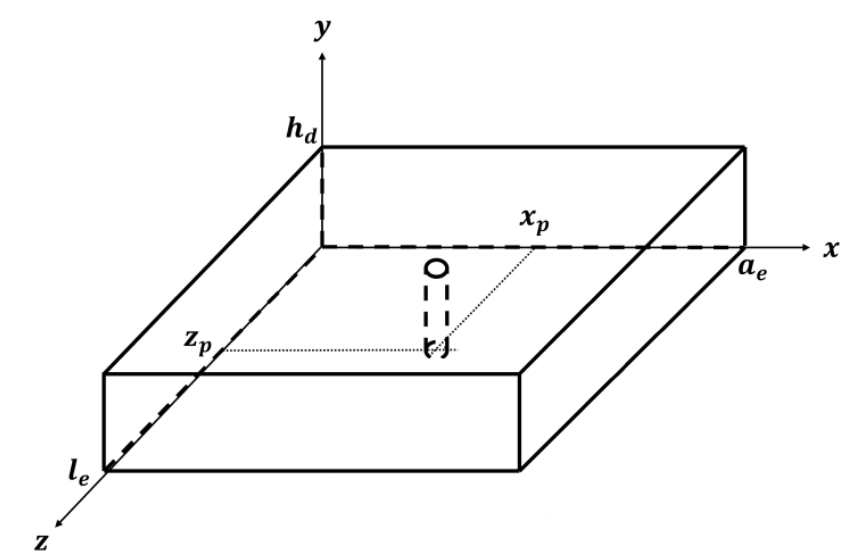

Fig.2. A thin metal post inserted in a resonant cavity in SIW technology.

where $h_{d}$ is the dielectric substrate thickness, $x_{p}$ and $z_{p}$ is the metal post position and $a_{e}$ is the effective width and $l_{e}$ is the effective length of the SIW cavity [11]-[16], given by

$$
\begin{aligned}
& a_{e}=a-1.08\left(\frac{d^{2}}{p}\right)+0.1\left(\frac{d^{2}}{a}\right) \\
& l_{e}=l-1.08\left(\frac{d^{2}}{p}\right)+0.1\left(\frac{d^{2}}{l}\right) .
\end{aligned}
$$

The resonance frequency of the unperturbed resonant cavity $\left(f_{o}\right)$ is given by

$$
f_{o}=\frac{c}{2 \sqrt{\varepsilon_{r}}} \sqrt{\frac{1}{a_{e}^{2}}+\frac{1}{l_{e}^{2}}}
$$

A fitting equation (4) was proposed to predict the resonance frequency of a square resonant cavity perturbed by a single metal post inserted through its internal volume and located at the center of the cavity $\left(x_{p}=a_{e} / 2\right.$ and $\left.z_{p}=l_{e} / 2\right)$, connecting bottom and upper walls, therefore causing its maximum fields perturbation and, consequently, the maximum frequency variation [18].

$$
f_{M}=\frac{5 c}{6 \sqrt{\varepsilon_{r}} a_{e}}
$$

The $f_{M}$ is the maximum resonance frequency achieved by a square resonant cavity operating at $\mathrm{TE}_{101}$ mode when perturbed by a single metal post located at its center. To obtain (4), three square SIW resonant cavities using feeding lines and taper lines were designed employing as dielectric substrates RO4003 ${ }^{\mathrm{TM}}$, RT/duroid $6006^{\mathrm{TM}}$ and RT/duroid $6010^{\mathrm{TM}}$, with a thickness of $0.635 \mathrm{~mm}$, from Rogers Corporation. Cavities with sides ranging from $20 \mathrm{~mm}$ to $40 \mathrm{~mm}$ were simulated in the High Frequency Structure Simulator (HFSS), demonstrating resonance frequencies ranging from $2 \mathrm{GHz}$ to $7 \mathrm{GHz}$. The metal post considered in all computational simulations has a diameter of $1 \mathrm{~mm}$ and was located at the center of the cavity. 
The several perturbed resonance frequencies obtained by computational simulations were tabulated, whereupon a factor $\varphi$ and hyperbole of first degree were considered to obtain an appropriating fitting, as given by

$$
f_{M}\left(a_{e}\right)=\varphi \frac{c}{\sqrt{\varepsilon_{r}} a_{e}} .
$$

The resulting resonance frequencies are presented in Fig. 3.

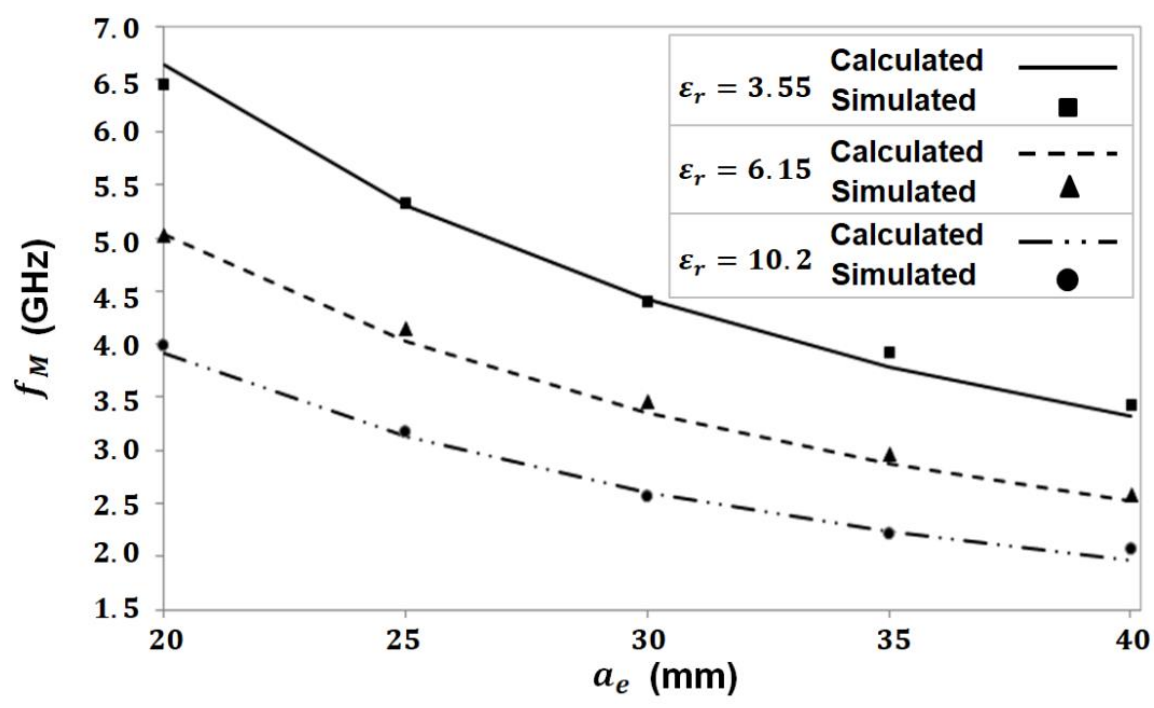

Fig.3. Calculated and simulated maximum resonance frequency.

The results presented in Fig. 3 show good agreement between simulated frequencies and the ones predicted by (4), considering dielectric substrates with dielectric constants of 3.35, 6.15 and 10.2. The ratio between $f_{M}$ and $f_{o}$ for a square resonant cavity using (3) and (4) is

$$
\frac{f_{M}}{f_{o}}=\frac{5}{3 \sqrt{2}} \approx 1.18,
$$

indicating that the maximum resonance frequency increases $18 \%$ in comparison with the unperturbed resonant cavity when a $1 \mathrm{~mm}$ diameter metal post is inserted at its center, connecting the upper and bottom walls. This frequency variation is due to the changes in the stored electric energy and magnetic energy caused by the metal post inserted in the cavity. According to [17], [20] and [22], it is mathematically described by

$$
\frac{f_{M}-f_{o}}{f_{o}}=\frac{\Delta W_{m}-\Delta W_{e}}{W_{m}+W_{e}},
$$

where $\Delta W_{m}$ and $\Delta W_{e}$ are the changes in the stored magnetic energy and electric energy, consecutively, after a shape modification and $W_{m}+W_{e}$ is the total energy stored in the cavity. The metal post reduces the cavity internal volume, modifying the electric and magnetic field distributions, therefore causing an increase of the frequency variation. If the metal post is moved from the center of the resonant cavity toward the sidewalls, the resonance frequency decreases, thus reducing the frequency variation. 


\section{B. Design of the tunable resonant cavity}

A square SIW resonant cavity was designed to resonate at $3.4 \mathrm{GHz}$ and $\mathrm{TE}_{101}$ mode. Inserting a metal post with a $1 \mathrm{~mm}$ diameter at the center of this cavity, a resonance frequency of $4.0 \mathrm{GHz}$ is predicted by (5). The designed tunable resonant cavity is fed by a $50 \Omega$ microstrip line and the impedance matching between the feeding line and the resonant cavity is performed by a tapered line. One end of the metal post is connected to the bottom wall of the cavity, and its other end can be left open or can be connected to the upper wall of the cavity using a switch. The present work uses a PIN diode and a jumper as switching elements.

According to [20] and [22], a metal post connected only at the bottom or upper wall does not significantly change the field distributions inside the resonant cavity; the resonance frequency of the cavity must thus be close to $3.4 \mathrm{GHz}$ in this condition. Therefore, considering (3), (4) and (5), the tunable resonant cavity was designed on a Rogers $\mathrm{RO} 4003 \mathrm{C}^{\mathrm{TM}}$ and its physical dimensions are presented in Fig. 1 and Table I.

TABLE I. PHYSICAL DIMENSIONS OF THE DESIGNED TUNABLE RESONANT CAVITY

\begin{tabular}{cccc}
\hline \multicolumn{4}{c}{ Physical dimension $(\mathbf{m m})$} \\
\hline $\boldsymbol{a}$ & 33.2 & $\boldsymbol{l}_{\mathbf{2}}$ & 14.0 \\
$\boldsymbol{d}$ & 1.00 & $\boldsymbol{w}_{\mathbf{2}}$ & 7.00 \\
$\boldsymbol{l}$ & 33.2 & $\boldsymbol{h}_{\boldsymbol{d}}$ & 0.635 \\
$\boldsymbol{p}$ & 2.54 & $\boldsymbol{a}_{\boldsymbol{p}}$ & 16.6 \\
$\boldsymbol{l}_{\mathbf{1}}$ & 11.8 & $\boldsymbol{l}_{\boldsymbol{p}}$ & 16.6 \\
$\boldsymbol{w}_{\mathbf{1}}$ & 1.12 & & \\
\hline
\end{tabular}

\section{Biasing Network}

Generally, to use PIN diodes as switching elements, a three-layer PCB implementation is necessary to separate the switching device biasing network from the microwave planar filter. In this paper, the biasing network was designed on a thin RT/duroid 5870 with a thickness of $254 \mu \mathrm{m}$, where this dielectric substrate is assembled on the upper wall of the resonant cavity. Figure 4 shows a description of the proposed tunable resonant cavity, where the dotted line denotes the cross-section for a better understanding.

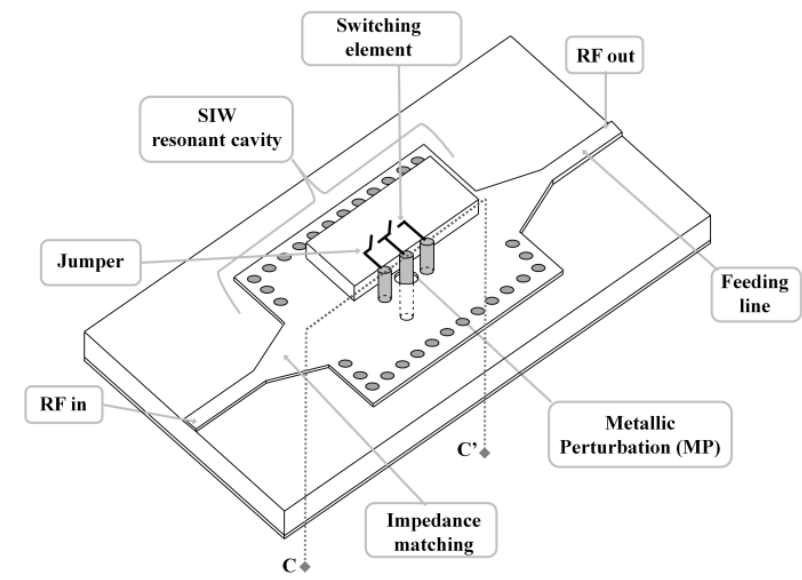

(a)

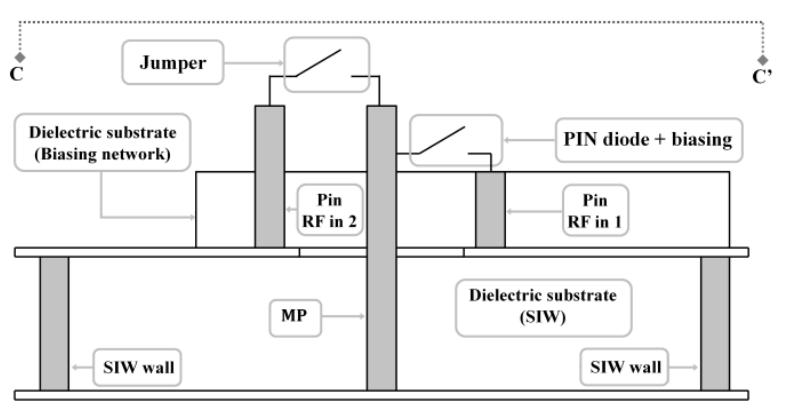

(b)

Fig.4. Proposed tunable resonant cavity: (a) Full view, (b) Cross-section CC'.

Brazilian Microwave and Optoelectronics Society-SBMO Brazilian Society of Electromagnetism-SBMag received 6 Sept 2019; for review 19 Sept 2019; accepted 20 Jan 2020 $\begin{array}{lll}\text { (C) } 2020 \text { SBMO/SBMag } & \text { (cc) BY } \\ \end{array}$ 
Two paths are considered to connect the metal post to the upper wall of the cavity. The first path uses the metal pin RF in 1 and a PIN diode switch to allow the microwave signal to flow from the upper wall to the metal post. The second path is established using a jumper and the metal pin RF in 2 . The jumper is considered to verify the performance of the biasing network used to bias the PIN diode. The connection using the jumper path is close to the ideal case when the metal post is directly soldered at the upper and bottom walls of the cavity, eliminating the imposed parasitic presence of the elements that compose the PIN switch.

Aiming to reduce the fabrication cost and the insertion losses, the PIN diode switch employs only one capacitor and transmission lines, replacing microwave-lumped components. The PIN diode switch uses the series configuration and was designed at $3.7 \mathrm{GHz}$, center frequency between $3.4 \mathrm{GHz}$ and 4.0 GHz. The bias voltage is connected to the PIN diode through a bias filter composed of a quarter-wavelength microstrip line with high characteristic impedance $\mathrm{Z}_{\mathrm{H}}=90 \Omega$ and an open quarterwavelength stub with $\mathrm{Z}_{\mathrm{L}}=20 \Omega$. The PIN diode switch is presented in Fig. 5 along with its circuit and computational model.
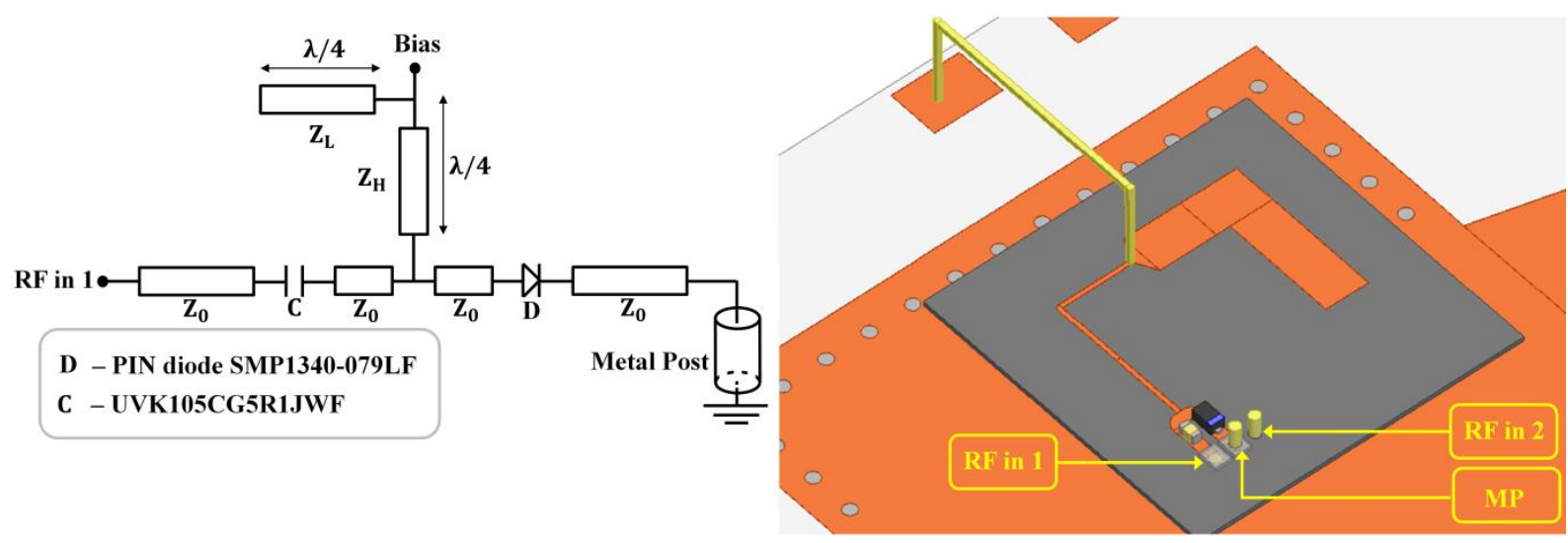

Fig.5. PIN diode switch.

In the series configuration, the switch is ON when the diode is forward-biased and OFF when it is reverse-biased. Bias voltages of $0.85 \mathrm{~V}$ and $-15 \mathrm{~V}$ were used for forward and reverse bias, respectively. The jumper ON state is close to a short circuit, while its OFF state is considered an open circuit. Table II shows the operation states.

TABLE II. Operation states of the tunable resonant cavity.

\begin{tabular}{ccccc}
\hline State & PIN diode & Jumper & $\boldsymbol{f}(\mathbf{G H z})$ & $\Delta \boldsymbol{f}(\mathbf{G H z})$ \\
\hline 1 & OFF & OFF & 3.4 & 0.00 \\
2 & ON & OFF & 4.0 & 0.60 \\
3 & OFF & ON & 4.0 & 0.60 \\
\hline
\end{tabular}

\section{EXPERIMENTAL AND SIMULATED RESULTS}

The tunable resonant cavity was fabricated and its performance was verified experimentally. The experimental results were compared to computational electromagnetic simulation results performed in the HFSS and theoretical results. A Vector Network Analyzer HP8722 was used to perform the electrical characterization. 

DOI: http://dx.doi.org/10.1590/2179-10742020v19i11881

Regarding the fabrication process, the SIW walls, metal post, RF in 1 and RF in 2 were implemented using metallic pins of low-cost pinhead connectors. Fig. 6 shows in detail the geometry of the hole in the resonant cavity which metal post goes. The metal post and RF in 1 are spaced by a distance of $1.27 \mathrm{~mm}$, as well the metal post and RF in 2 .

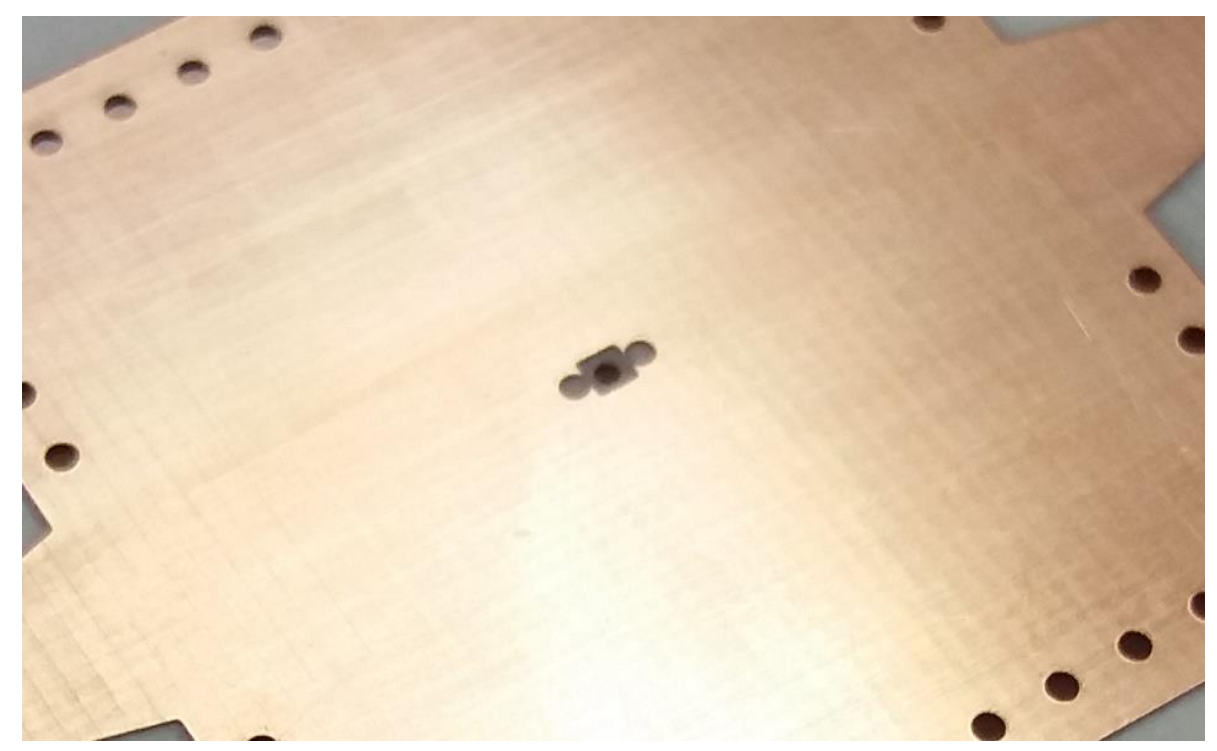

Fig. 6. Assembling detail of metal post, RF in 1 and RF in 2.

The metal post was soldered at the bottom wall and was left as open via at the upper wall, the RF in 1 and RF in 2 were soldered at the top wall, as shown by Fig. 4. To perform good soldering and keep the correct distance between metal post, RF in 1 and RF in 2, the plastic that involves the pinhead connectors was kept during the soldering processes and removed after it. The finished fabricated structure and the measurement setup used to experimentally obtain the S parameters are presented by Fig. 7.

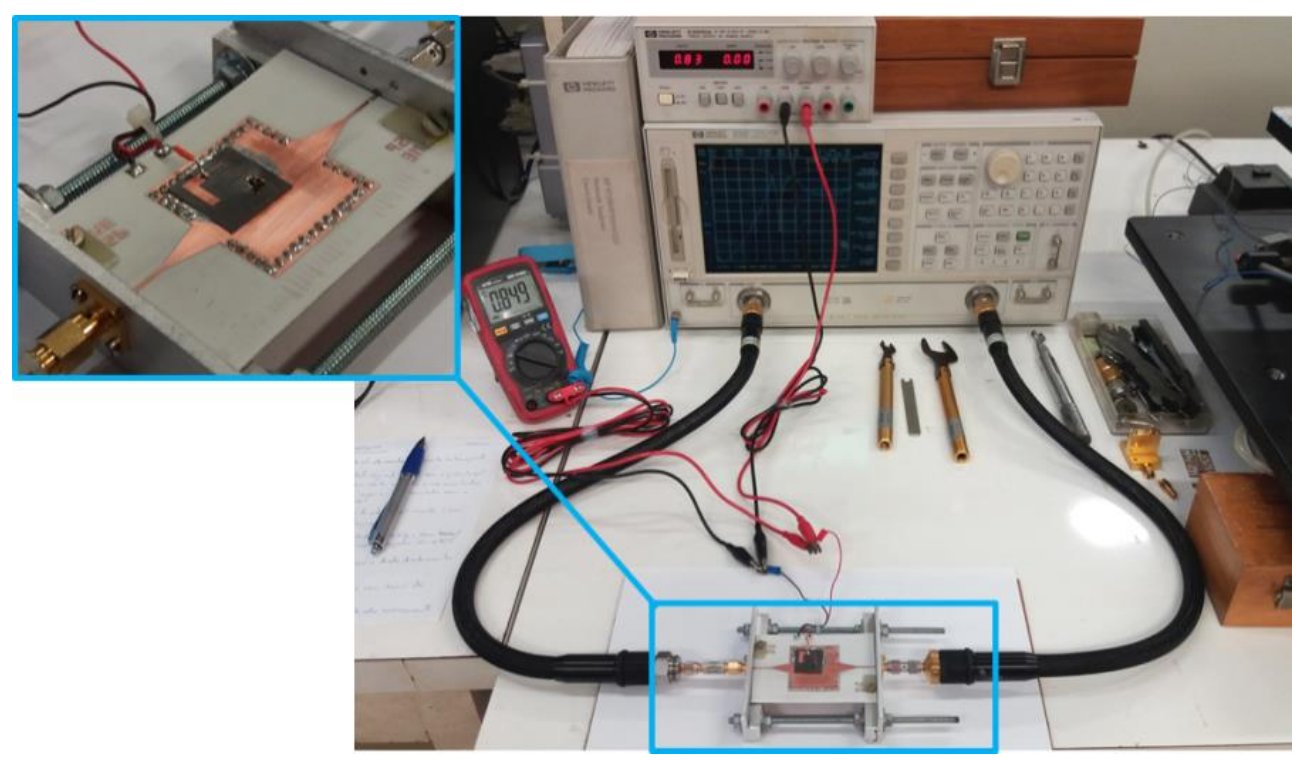

Fig. 7. Photography of the fabricated tunable resonant cavity and its measurement setup. 
Figure 8 shows the $S_{11}$ parameters of the unperturbed resonant cavity and the electric field plotted on the ground plane at $3.40 \mathrm{GHz}$. In this case, the metal post was not inserted inside its internal volume.

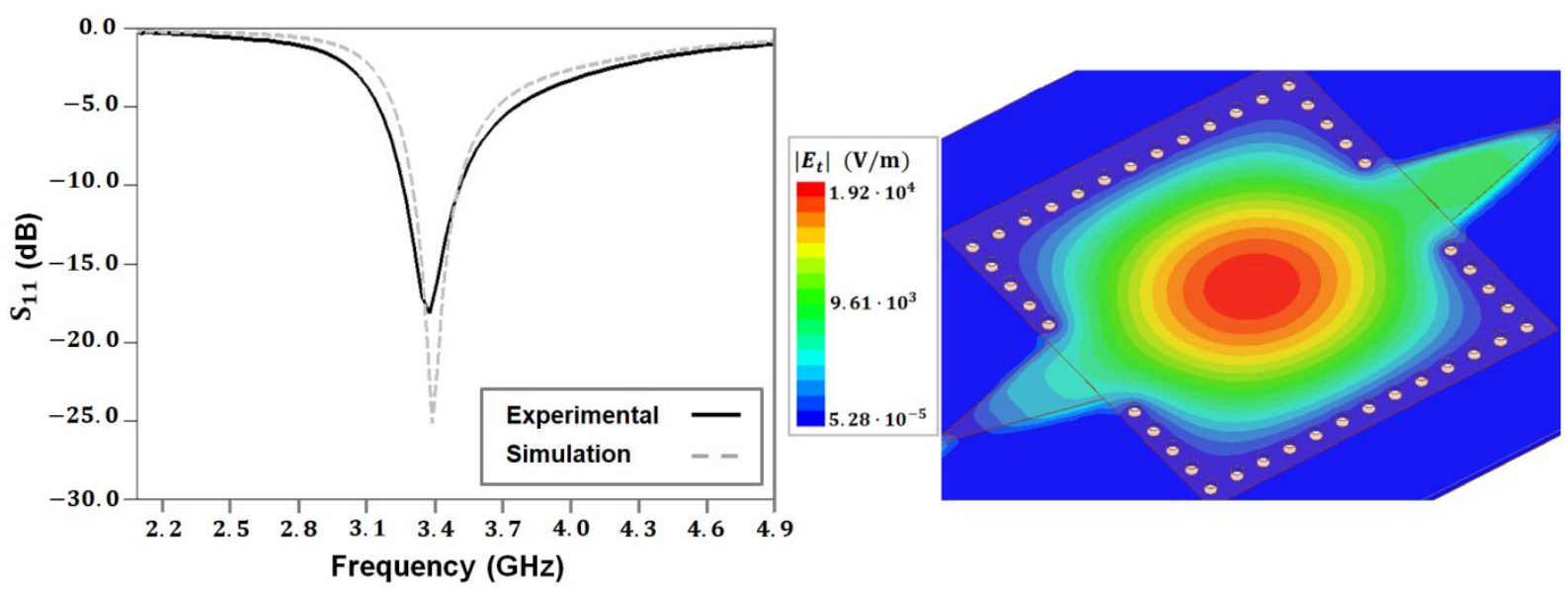

Fig. 8. Simulation and experimental results of the unperturbed resonant cavity.

Figure 8 shows a measured resonance frequency of $3.37 \mathrm{GHz}$, resulting in an error of $0.88 \%$ in comparison with the calculated value of $3.40 \mathrm{GHz}$ and the simulated value of $3.40 \mathrm{GHz}$. As expected for the $\mathrm{TE}_{101}$ mode, the maximum electric field occurs at the center of the resonant cavity. For this case, there is no metal post allocated in the position of maximum field imposing $\boldsymbol{E}_{\boldsymbol{t}}=0$. It is important to verify this field distribution to make comparisons with the field distributions related to the three operation states presented by Table II.

According to Table II, state 1 is achieved when the PIN diode is OFF and the jumper is OFF. The $\mathrm{S}$ Parameters related to state 1 and the electric field plotted on the ground plane are presented in Fig. 9.

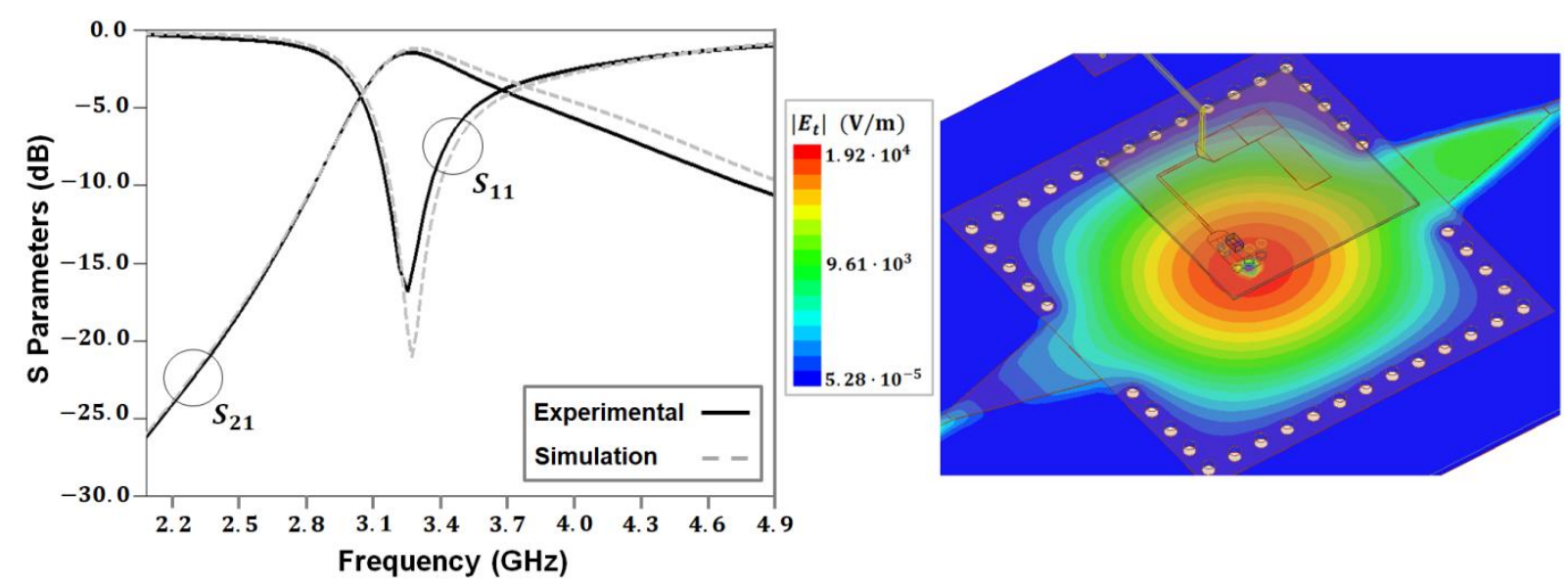

Fig. 9. Simulation and experimental results of state 1.

Figure 9 shows a measured resonance frequency of $3.23 \mathrm{GHz}$, resulting in an error of $1.22 \%$ in comparison with the simulated value of $3.27 \mathrm{GHz}$. The electric field plotted on the ground plane at $3.27 \mathrm{GHz}$ shows that the open metal post does not change significantly change its distribution as expected and predicted by [20] and [22], close to the $\mathrm{TE}_{101}$ unperturbed mode. 
According to Table II, state 2 is achieved when the PIN diode is ON and the jumper is OFF. The $\mathrm{S}$ Parameters related to state 2 and the electric field plotted on the ground plane are presented in Fig. 10.
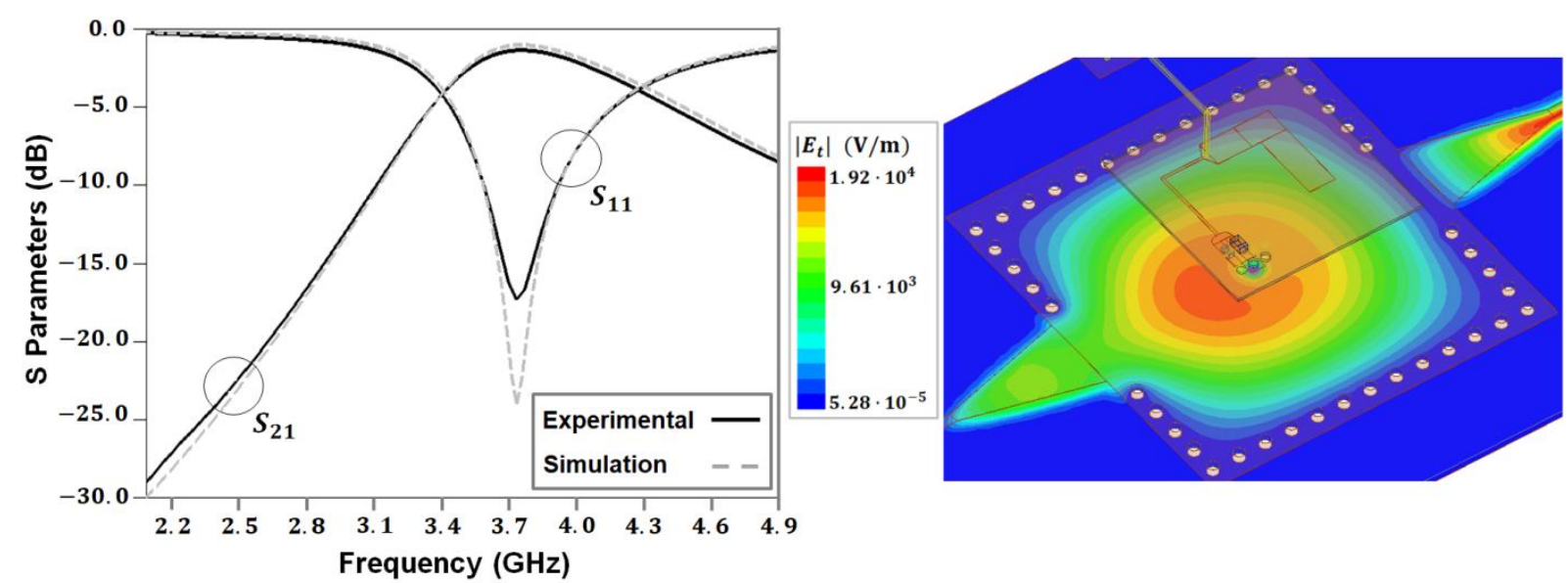

Fig. 10. Simulation and experimental results of state 2 .

Figure 10 shows a measured resonance frequency of $3.71 \mathrm{GHz}$, resulting in an error of $0.54 \%$ in comparison with the simulated value of $3.73 \mathrm{GHz}$ and a frequency increase of $0.48 \mathrm{GHz}$ compared to state 1 . The electric field plotted at $3.73 \mathrm{GHz}$ shows that the boundary condition $\boldsymbol{E}_{\boldsymbol{t}}=0$ is imposed when the metal post is connected at the bottom and upper walls, and the resonant cavity has its $\mathrm{TE}_{101}$ mode perturbed, changing the position of the maximum electrical field and causing a change in the stored electric energy and magnetic energy.

According to Table II, state 3 is achieved when the PIN diode is OFF and the jumper is ON. The $\mathrm{S}$ Parameters related to state 3 and the electric field plotted on the ground plane are presented in Fig. 11 .

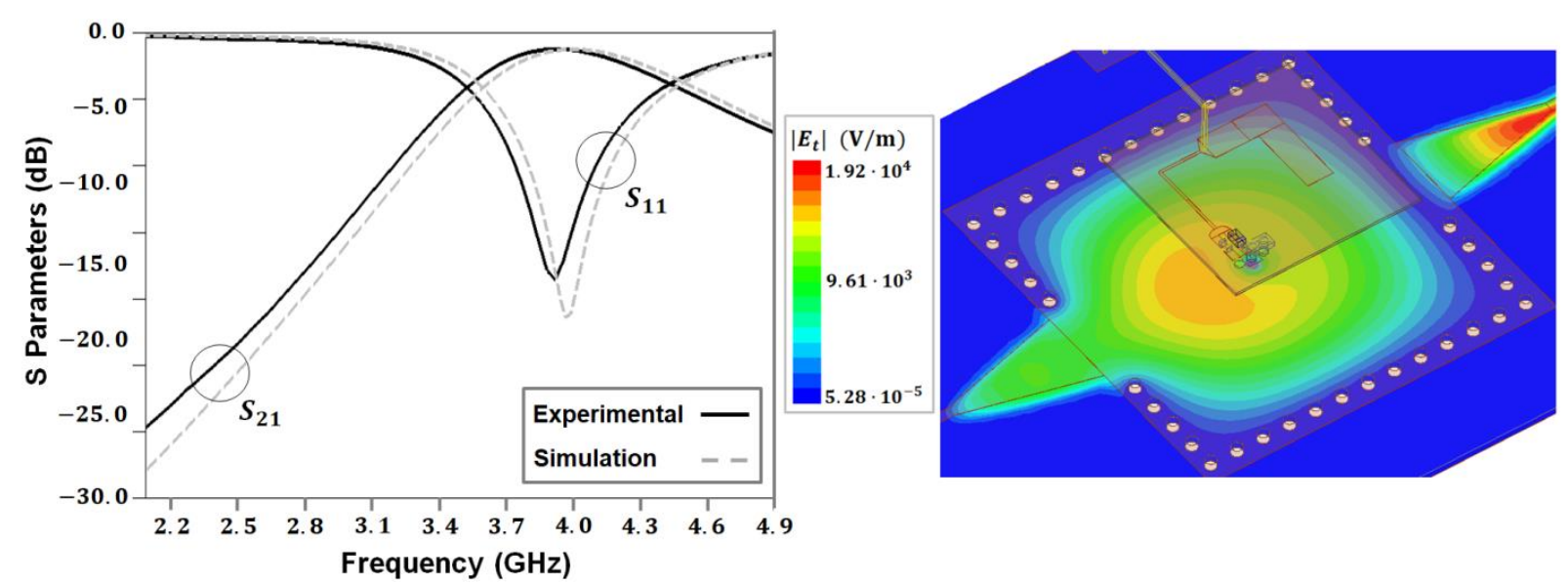

Fig. 11. Simulation and experimental results of state 3.

Figure 11 shows a measured resonance frequency of $3.86 \mathrm{GHz}$, resulting in an error of $2.77 \%$ in comparison with the simulated value of $3.97 \mathrm{GHz}$, an error of $3.50 \%$ in comparison with the calculated value of $4.0 \mathrm{GHz}$, and a frequency increase of $0.63 \mathrm{GHz}$ compared to state 1 . The electric field plotted at $3.97 \mathrm{GHz}$ also shows that the boundary condition $\boldsymbol{E}_{\boldsymbol{t}}=0$ is achieved when the metal post is connected at the bottom and upper walls, and the resonant cavity has the $\mathrm{TE}_{101}$ mode perturbed. This situation is closer to the ideal case when the metal post is directly connected at the upper and bottom walls. Fig. 12 shows all the $S_{11}$ measured parameters. 


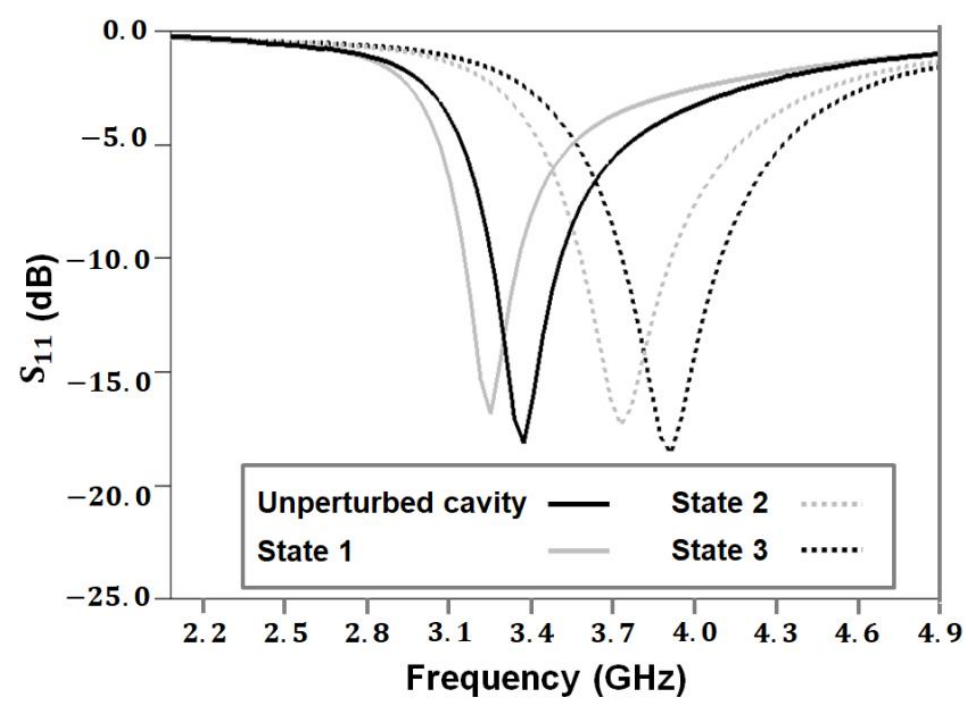

Fig. 12. $\mathrm{S}_{11} \mathrm{x}$ frequency measured curves.

Figure 12 shows that the resonance frequency of the cavity can be tuned by $19.5 \%$ using the jumper to act as an ideal switch. A tuning range of $14.9 \%$ was obtained using the PIN switch, having been reduced by the no ideal behavior of the PIN diode at the reverse and forward bias conditions. These tuning ranges are compatible with the $18 \%$ range predicted by (5). All the states show good agreement for $S_{11}$ and $S_{21}$, validating the proposed fitting equation and the biasing network employing transmissions lines and a single capacitor assembled on the upper wall of the resonant cavity in SIW technology. Some comparisons between this and previous works are summarized in Table III.

TABLE III. Comparison and performance summary.

\begin{tabular}{|c|c|c|c|}
\hline Reference & Switch type & Insertion Loss and Frequency & $\Delta f(\mathbf{G H z})$ \\
\hline This work & $\begin{array}{l}\text { PIN diode switch with } \\
\text { transmission lines }\end{array}$ & $\begin{array}{l}1.47 \mathrm{~dB} \text { at } 3.27 \mathrm{GHz} \text { (state } 1) \\
1.38 \mathrm{~dB} \text { at } 3.71 \mathrm{GHz} \text { (state 2) }\end{array}$ & 0.44 \\
\hline [17] & $\begin{array}{l}\text { Surface actuator } \\
\text { (as described) }\end{array}$ & $\begin{array}{c}2.13 \mathrm{~dB} \text { at } 9.97 \mathrm{GHz} \text { (state off) } \\
1.88 \mathrm{~dB} \text { at } 9.47 \mathrm{GHz} \text { (state on) }\end{array}$ & 0.50 \\
\hline [18] & $\begin{array}{l}\text { PIN diode switch with } \\
\text { lumped components }\end{array}$ & $\begin{array}{l}3.8 \mathrm{~dB} \text { at } 1.55 \mathrm{GHz} \text { (state } 1) \\
4.4 \mathrm{~dB} \text { at } 2.00 \mathrm{GHz} \text { (state } 6)\end{array}$ & 0.45 \\
\hline
\end{tabular}

\section{CONCLUSION}

A tunable resonant cavity in SIW technology was proposed and fabricated to operate in the range from $3.3 \mathrm{GHz}$ to 4.2 , related to the spectrum considered for the $5 \mathrm{G}$ network. An unperturbed resonant cavity in SIW technology was designed to operate at $3.4 \mathrm{GHz}$, whereupon a metal post and switches were used to increase the resonance frequency. To achieve different states, a PIN diode, a jumper and a single metal post precisely allocated inside the internal volume of the resonant cavity were used, connecting and disconnecting the upper and the bottom wall. A fitting equation was proposed to predict the maximum resonance frequency of a square SIW resonant cavity perturbed by a single metal post inserted inside its internal volume. An error of 3.50\% in comparison with the measured value was obtained for the resonance frequency using the proposed fitting equation. Aiming to reduce the fabrication cost and the insertion losses of the dual-band resonant cavity, a new low-cost biasing 
network was designed on a thin dielectric substrate using transmission lines and a single capacitor. Experimental and electromagnetic simulation results showed an error of less than $1.3 \%$ for states 1 and 2 and $2.8 \%$ for state 3 . The proposed dual-band resonant cavity can be applied as a narrowband filter to frequencies below $6 \mathrm{GHz}$ in receivers and transmitters to be used in $5 \mathrm{G}$ networks. The proposed biasing network can be applied to other types of filters based on resonant cavities and waveguides in SIW technology, resulting in a compact and low-cost structure, besides reducing the insertion losses.

\section{ACKNOWLEDGMENT}

The authors would like to acknowledge the Rogers Corporation for providing the substrate samples.

\section{REFERENCES}

[1] A. Wortmann, A, B. Combemale, O. Barais, "A systematic mapping study on modelling for industry 4.0," in Int. Conf. on Model Driven Eng. Languages and Syst., Austin, USA, Sept. 2017, pp. 281-291.

[2] F. Bonavolontà, A. Tedesco, R. S. L. Moriello, et al, "Enabling wireless technologies for industry 4.0: State of the Art," in Int. Workshop on Measurement and Networking, Naples, Italy, Sept. 2017, pp. 1-5.

[3] M. Wollschlaeger, T Sauter, J. Jasperneite, "The Future of Industrial Communication: Automation Networks in the Era of the Internet of Things and Industry 4.0," IEEE Ind. Electron. Mag., vol. 11, no. 1, pp. 17-27, Mar. 2017.

[4] C. Leyh, S. Martin, T. Schäffer, "Industry 4.0 and Lean Production - A Matching Relationship? An analysis of selected Industry 4.0 models," in Federated Conf. on Comput. Sci. and Inform. Syst., Prague, Czech Republic, Sept. 2017, pp. 989993.

[5] W. Ejaz, M. Ibnkahla, "Multiband Spectrum Sensing and Resource Allocation for IoT in Cognitive 5G Networks," IEEE Internet Things J., vol. 5, no. 1, pp.150-163, Feb. 2018.

[6] D. Schinianakis, "Alternative Security Options in the 5G and IoT Era," IEEE Circuits Syst. Mag., vol. 17, no. 4, pp. 6-28. Fourthquarter 2018.

[7] E. Ezhilarasan, M. Dinakaran, "A review on Mobile Technologies: 3G, 4G and 5G," in Int. Conf. on Recent Trends and Challenges in Computational Models, Tindivanam, India, Feb. 2017, pp. 369-373.

[8] T. Sharma, K. Ritesh, N. Chauhan, et al, "Analogous study of 4G and 5G," in Int. Conf. on Computing for Sustainable Global Develop., New Delhi, India, Mar. 2016, pp. 2137-2140.

[9] I. Belikaidis, A. Georgakopoulos, E. Kosmatos, et al, "Management of 3.5-GHz Spectrum in 5G Dense Networks: A Hierarchical Radio Resource Management Scheme,” IEEE Veh. Technol. Mag., vol. 13, no. 2, pp. 57-64, Jun. 2018.

[10] J. Lee, E. Tejedor, K. Ranta-aho, et al, "Spectrum for 5G: Global Status, Challenges, and Enabling Technologies," IEEE Commun. Mag., vol. 5, pp. 12-18, Mar. 2018.

[11] Y. Cassivi, L. Perregrini, P. Arcioni, P, et al, "Dispersion characteristics of substrate integrated rectangular waveguide," IEEE Microw. Compon. Lett., vol. 12, no. 9, pp. 333-335, Sept. 2002.

[12] M. Bozzi, A. Georgiadis, K. Wu, "Review of substrate-integrated waveguide circuits and antennas". IET Microw., Antennas Propag., vol. 5, no. 8, pp. 909-920, Jun. 2011

[13] X. Chen, K. Wu, "Substrate Integrated Waveguide Filter: Basic Design Rules and Fundamental Structure Features," IEEE Microw. Mag., vol. 15, no. 5, pp. 108-116, July-Aug. 2014.

[14] X. Chen, K. Wu, "Substrate Integrated Waveguide Filters: Design Techniques and Structure Innovations," IEEE Microw. Mag., vol. 15, no. 6, pp. 121-133, Sept.-Oct. 2014.

[15] X. Chen, K. Wu, "Substrate Integrated Waveguide Filters: Practical Aspects and Design Considerations," IEEE Microw. Mag., vol. 15, no. 7, pp. 75-83, Nov.-Dec. 2014.

[16] R. C. Caleffo, "New Design Procedure to Determine the Taper Transition for Impedance Matching Between Microstrip Line and SIW Component," J. of Microw. Optoelectronics and Electromagn. Appl., vol. 15, no. 3, pp. 247-260, JulySep. 2016.

[17] J. C. Bohorquez, B. Potelon, C. Person, et al, "Reconfigurable Planar SIW Cavity Resonator and Filter," in Int. Microw. Symp. Dig., San Francisco, USA, Jun. 2006, pp. 947-950.

[18] M. Armendariz, V. Sekar, K. Entesari, "Tunable SIW Bandpass filters with PIN diodes," in Eur. Microw. Conf., Paris, France, Sept. 2010, pp. 830-833.

[19] R. C. Caleffo, F. S. Correra, "Liquids electrical characterization sensor using a hybrid SIW resonant cavity," Microw. and Opt. Technol. Lett., vol. 60, no. 2, pp. 445-449, Jan. 2018.

[20] D. M. Pozar, Microwave Engineering, 4th ed. New York, NY, USA: John Wiley \& Sons, Inc., 2011.

[21] S. Ramo, J. R. Whinnery, T. V. Duzer, Fields and waves in communication electronics, 3rd ed. New York, NY, USA: John Wiley \& Sons, Inc., 1993.

[22] R. G. Carter, "Accuracy of microwave cavity perturbation measurements," IEEE Trans. Microw. Theory Techn., vol. 49, no. 5, pp. 918-923. May 2001. 